\title{
Transição epidemiológica e o estudo de carga de doença no Brasil
}

\author{
Epidemiological transition and the study \\ of burden of disease in Brazil
}

Joyce Mendes de Andrade Schramm 1

Andreia Ferreira de Oliveira 2

Iúri da Costa Leite 1

Joaquim Gonçalves Valente 1

Ângela M aria Jourdan Gadelha 1

M argareth Crisóstomo Portela 3

M ônica Rodrigues Campos 4

\footnotetext{
1 Departamento de Epidemiologia e M étodos Quantitativos da Escola Nacional de Saúde Pública, Fiocruz.

Rua Leopoldo Bulhões 1480, M anguinhos, 21041-210,

Rio de Janeiro RJ. Joyce@ensp.fiocruz.br 2 Secretaria M unicipal de Saúde do Rio de Janeiro.

3 Departamento de

Administração e

Planejamento da Escola

Nacional de Saúde Pública,

Fiocruz.

4 Departamento de

Ciências Sociais da Escola

Nacional de Saúde Pública,

Fiocruz.
}

Abstract In Brazil, the epidemiological transition has not followed the model experienced by most developed countries. There is coexistence of old and new health problems, where despite the predominance of the chronical and degenerative diseases, the communicable ones still play an important role. In this study the differentials in relation to the epidemiological pattern are described not only for the country as a whole, but also for its major regions, using the Disability Adjusted Life Years (DALY), the health indicator of the studies of burden of disease. Amongst the main results found we stand out that the group of chronical and degenerative di seases is responsible for $66,3 \%$ of the national burden of disease; $23,5 \%$ are responded by the communicable diseases, perinatal and maternal conditions and nutritional deficiencies; and $10,2 \%$ is due to the injuries. The use of DALY permits the identification of health priorities based on the epidemiological profile, making easier the process of decision make and the use of resources by the managers.

Key words Epidemiological transition, Burden of di sease, DALY, N on-communicable diseases
Resumo No Brasil, a transição epidemiológica não tem ocorrido de acordo com o modelo experimentado pela maioria dos países desenvolvidos. Velhos e novos problemas em saúde coexistem, com predominância das doenças crônico-degenerativas, embora as doenças transmissíveis ainda desempenhem um papel importante. N este estudo, os diferenciais, em relação ao padrão epidemiológico, são descritos para o Brasil e grandes regiões, para o indicador de saúde dos estudos da carga de doença, o DALY. Entre os principais resultados encontrados, para o Brasil, destaca-se que o grupo das doenças não-transmissíveis, infecciosas/parasitárias/maternas/perinatais/nutricionais, e das causas externas representaram, respectivamente, $66,3 \%, 23,5 \%$ e $10,2 \%$ da carga total de doença estimada. A utilização do indicador DALY propicia a identificação de prioridades em função do perfil epidemiológico, facilitando a tomada de decisões e destinação adequada de recursos por parte dos gestores.

Palavras-chave Transição epidemiológica, Carga de doença, DALY, D oenças não-transmissíveis 


\section{Introdução}

Entende-se por transição epidemiológica as mudanças ocorridas no tempo nos padrões de morte, morbidade e invalidez que caracterizam uma população específica eque, em geral, ocorrem em conjunto com outras transformações demográficas, sociais e econômicas ( 0 mram, 2001; Santos-Preciado et al., 2003). 0 processo engloba três mudanças básicas: substituição das doenças transmissíveis por doenças não-transmissíveis e causas externas; deslocamento da carga de morbi-mortalidade dos grupos mais jovens aos grupos mais idosos; e transformação de uma situação em que predomina a mortalidade para outra na qual a morbidade é dominante. A definição da transição epidemiológica deve, assim, ser considerada componente de um conceito mais amplo apresentado por Lerner (1973) como transição da saúde, que inclui elementos das concepções e comportamentos sociais, correspondentes aos aspectos básicos $d a$ saúde nas populações humanas.

A transição da saúde pode de dividir em dois elementos principais: de um lado, encontra-se a transição das condições de saúde (referindo-se às mudanças na freqüência, magnitude e distribuição das condições de saúde, expressas através das mortes, doenças e incapacidades) e de outro, a resposta social organizada a estas condições que se instrumenta por meio dos sistemas de atenção à saúde (transição da atenção sanitária), determinada em grande medida pelo desenvolvimento social, econômico e tecnológico mais amplo (Frenk et al., 1991).

Segundo Chaimowicz (1997), existe uma correlação direta entre os processos de transição epidemiológica e demográfica. A princípio, o declínio da mortalidade concentra-se seletivamente entre as doenças infecciosas e tende a beneficiar os grupos mais jovens da população, que passam a conviver com fatores de risco associados às doenças crônico-degenerativas e, na medida em que cresce o número de idosos e aumenta a expectativa de vida, as doenças nãotransmissíveis tornam-se mais freqüentes.

A modificação no perfil de saúde da população em que as doenças crônicas e suas complicações são prevalentes resulta em mudanças no padrão de utilização dos serviços de saúde e no aumento de gastos, considerando a necessidade de incorporação tecnológica para o tratamento das mesmas. Estes aspectos ocasionam importantes desafios e a necessidade de uma agenda para as políticas de saúde que possam dar conta das várias transições em curso. A escalada tecnológica, o modelo hospitalocêntrico ainda vigente, a pouca valorização na educação médica e de outros profissionais em relação aos aspectos referentes à promoção e prevenção, a necessidade de novas instâncias de cuidados (além do hospitalar e do ambulatorial clássicos), as marcantes deficiências qualitativas e quantitativas da força de trabalho em saúde e o desenvolvimento de programas e políticas custo-efetivas são elementos a serem considerados no desenvolvimento dos futuros modelos tecno-assistenciais em saúde (Popkin, 1994; Goulart, 1999).

No Brasil, a transição epidemiológica não tem ocorrido de acordo com o modelo experimentado pela maioria dos países industrializados e mesmo por vizinhos latino-americanos como o Chile, Cuba e Costa-Rica. Há uma superposição entre as etapas nas quais predominam as doenças transmissíveis e crônico-degenerativas; a reintrodução de doenças como dengue e cólera ou o recrudescimento de outras como a malária, hanseníase e leishmanioses indicam uma natureza não-unidirecional denominada contra-transição; o processo não se resolve de maneira clara, criando uma situação em que a morbi-mortalidade persiste elevada para ambos os padrões, caracterizando uma transição prolongada; as situações epidemiológicas de diferentes regiões em um mesmo país tornam-se contrastantes (polarização epidemiológica) (Frenk et al., 1991). Além disso, o enveIhecimento rápido da população brasileira a partir da década de 1960 faz com que a sociedade se depare com um tipo de demanda por serviços médicos e sociais outrora restrita aos países industrializados. 0 Estado, ainda às voltas em estabelecer o controle das doen ças transmissíveis e a redução da mortalidade infantil, não foi capaz de desenvolver e aplicar estraté gias para a efetiva prevenção e tratamento das doenças crônico-degenerativas e suas complicações levando a uma perda de autonomia e qualidade de vida (Chaimowicz, 1997).

O conceito de transição epidemiológica tem merecido críticas pelo fato de a transformação dos padrões de saúde não obedecer aos mesmos parâmetros na seqüência, intensidade e velocidade, em diferentes regiões. Para Possas (2001), a heterogeneidade das sociedades contemporâneas impõe um padrão de risco de tênues fronteiras, a saber, os espaços urbano/rural e selva se interconectam, e riscos e patologias modernas arcaicas se sobrepõem. 
O utra principal debilidade do esquema teórico da transição epidemiológica seria a de enfatizar a tecnologia médica como principal alternativa interveniente no curso da transição, desconsiderando o papel que as variáveis econômicas e sociais desempenham neste processo (Barreto \& Carmo, 1995; Barreto et al., 1993).

Este trabalho tem por objetivo descrever os diferenciais existentes quanto ao padrão epidemiológico no Brasil e grandes regiões, utilizando como indicador o DALY (Disability Adjusted Life of Years - Anos de Vida Perdidos Ajustados por Incapacidade).

\section{Material e métodos}

O DALY é um indicador que procura medir simultaneamente o impacto da mortalidade e dos problemas de saúde que afetam a qualidade de vida dos indivíduos. O DALY mede os anos de vida perdidos seja por morte prematura (YLL -Years of Life Lost - Anos de vida perdidos por morte prematura) ou incapacidade (YLD - Years Lived with Disability - Anos de vida vividos com incapacidade) em relação a uma esperança de vida ideal cujo padrão utilizado foi o do Japão, país com maior esperança de vida ao nascer do mundo ( 80 anos para homense 82,5 anos para mulheres). A metodologia propõe a utilização da tábua de mortalidade desenvolvida por Coale \& Guo (1989), na qual a esperança de vida é calculada para cada idade exata. A utilização de um mesmo padrão para todos os países é importante para garantir a comparabilidade dos resultados. Para que os anos perdidos por morte prematura e anos vividos com incapacidade possam ser adicionados é preciso criar uma escala associando pesos à mortalidade e às doenças e seqüelas. Neste sentido, o DALY está ancorado em uma escala de saúde que varia entre zero e um, onde zero significa o estado de plena saúde e um é a morte, o pior estado de saúde possível. Ressalte-se que atribuir peso às doenças ou seqüelas não implica dizer que a vida de seus portadores tenha menos valor do que a vida de indivíduos em perfeita saúde (Nord et al., 1999). Os pesos utilizados pelo Estudo da Carga de Doença do Brasil foram aqueles sugeridos pela OM S em suas publicações segundo sexo, faixa etária e, separadamente, segundo tratamento ou não da doença.

A metodologia do estudo da Carga de Doença propõe dois ajustes no cálculo de cada um desses componentes (YLL eYLD). 0 primeiro ajuste é feito por meio da incorporação de uma função de ponderação de idade que atribui pesos menores aos anos perdidos no início e fim do ciclo da vida - crianças e idosos ( M urray \& Lopez, 1996a; 1996b). Este ajuste não foi utilizado no estudo brasileiro, considerando-se as críticas que têm sido feitas ao uso da ponderação de idade, principalmente em relação à equanimidade do método (Arnand \& H anson, 1997; Bobadilla, 1996). O segundo ajuste refere-se à incorporação de uma taxa de desconto em relação aos anos perdidos no futuro, objetivando estimar os anos de vida perdidos no presente. Esta prática tem sido muito comum nos estudos em economia da saúde (D asgupta, 1972; Layard $\&$ Gleister, 1994). 0 desconto de futuros benefícios é praticado de forma padrão em análises econômicas e refere-se a uma prática de atribuir um valor maior ou menor no futuro quando comparado ao presente (Lind et al., 1982). No Estudo de Carga de Doença no Brasil optou-se por incorporar a taxa de desconto de 3\% ao ano, taxa definida em painel de experts na área de economia da saúde (Gold et al., 1996).

\section{Fontes de dados}

Utilizou-se a população residente, em 1998, estimada pelo IBGE, por sexo e faixa etária para cada Unidade da Federação (UF). Para obtenção dos óbitos utilizou-se o Sistema de M ortalidade para o ano de 1998 (último ano cujos dados estavam disponíveis). Procedeu-se à correção do sub-registro de óbitos utilizando-se técnicas indiretas (Gadelha et al., 2002). Os códigos-lixo, códigos inespecíficos que não permitem a classificação precisa da causa de óbito, foram redistribuídos, assim como as causas mal definidas (Gadelha et al., 2002). Nas estimativas dos parâmetros clínico-epidemiológicos (incidência, prevalência, duração e remissão) por UF, sexo e faixa etária, utilizaram-se as informações de morbidade de bancos de dados públicos que estão disponíveis no sistema Datasus ou que foram cordialmente cedidos por órgãos públicos/ministeriais.

Para obtenção dos referidos parâmetros foi realizada ampla revisão da literatura e de base de dados informatizados (via internet), definindo-se al guns critérios na seleção dos materiais quais sejam: priorização de dados nacionais; melhor desagregação dos parâmetros (segundo UF, sexo e faixa etária); ano-base de 
informação: 1998; ordem de prioridades das fontes bibliográficas/dados secundários ( 10 dados oficiais, 2o publicações/periódicos indexados, 3 o outras fontes) e priorização de grupos de pesquisa ou autores considerados referência na especialidade. Também recorreu-se a fontes de "literatura fugidia": capítulos de livro, anais de conferências/congressos, relatórios governamentais ou estudos não-publicados, de difícil identificação e obtenção, assim como consultas a experts. Todo o levantamento bibliográfico foi armazenado em um banco de dados e a documentação do protocolo de revisão normatizada por agravo/seqüela registrada em um documento denominado "diário de bordo".

\section{Resultados}

Para efeito de apresentação dos resultados do estudo, os agravos são agrupados em três grandes grupos: Grupo I - D oenças Infecciosas e Parasitárias, Causas Perinatais, Causas M aternas e Doenças Endócrinas; Grupo II - Doenças Não-Transmissíveis; Grupo III - Causas Externas. Apresentaremos inicialmente os resultados referentes à fração do DALY que corresponde aos anos de vida perdidos por morte prematu$\mathrm{ra}(\mathrm{YLL})$.

As informações referentes à distribuição proporcional do YLL, em ambos os sexos, segundo grupos de causa e grandes regiões, encontram-se na tabela 1.0 bserva-se que dentro do Grande Grupo I as doenças infecciosas/parasitárias e condições do período perinatal apresentam-se em destaque. Para ambas as situações, as regiões Norte e Nordeste apresentaram maior concentração (12\%-13\% e 18\%$14 \%$, respectivamente) em comparação com as regiões Sul/Sudeste (6\%-8\% e 7\%-7\%, respectivamente), sendo que a região Centro-0 este apresentou uma concentração intermediária entre os binômios N orte-N ordeste e SudesteSul. Ainda no Grupo I, apenas as infecções respiratórias se destacaram com $5 \%$ dos YLL, no Brasil como um todo, tendo oscilado, nas grandes regiões, de $4 \%$ a $6 \%$. No Grupo II, destacam-se as doenças cardiovasculares com $24 \%$ e o câncer com 12\%. No grupo das doenças cardiovasculares, as doenças isquêmicas do coração $(9,3 \%)$, juntamente com as doenças cerebrovasculares (Acidente Vascular Cerebral - 1o ataque - $8,1 \%$ ), se situam entre as duas primeiras causas de anos de vida perdidos por morte prematura no Brasil.
Destacam-se ainda as doenças do aparelho digestivo, com 7\% e as doenças respiratórias crônicas, com 5\%. O câncer apresenta uma concentração maior na região Sul, com 16\%, e menor nas regiões N orte, N ordeste e CentroOeste, com 10\%-11\%. As doenças cardiovasculares ocuparam o primeiro lugar em relação aos anos de vida perdidos por morte prematura no conjunto das doenças não-transmissíveis. Para o grupo III, para todas as regiões, o número de YLL foi maior nas causas não intencionais do que nas intencionais, exceto no Sudeste (onde houve inversão no ranking) e N ordeste, onde as proporções se equivaleram.

Tomando-se os resultados da tabela 2 , que apresenta as taxas e a distribuição proporcional do YLD segundo grupo de causas, sexo e grandes regiões, verifica-se que os YLD para o Brasil segundo os Grandes Grupos I, II e III foram respectivamente $19,8 \%, 74,7 \%$ e $5,5 \%$. As doenças infecciosas (Grande Grupo I) constituem uma causa importante de perda de YLD para o Brasil como um todo, sendo maior a proporção na região Norte $(14,1 \%)$ e N ordeste $(12,1 \%)$; intermediária, na região Centro-0 este $(7,9 \%)$ e menor nas regiões Sudeste $(6,3 \%)$ e Sul (6,9\%). No Grande Grupo III, das causas externas, chama a atenção os acidentes que representaram 5,1\% dos YLD por todas as causas com pouca variação inter-regional. As doenças neuropsiquiátricas, incluídas no Grande Grupo II, das doenças não-transmissíveis, foram responsáveis por mais de um terço (34\%) de toda a morbidade calculada para 0 Brasil. Vale ressaltar a importância que esse grupo de doenças assume em relação à morbidade, quando se compara com a mortalidade (tabelas 1 e 2).

A tabela 3 apresenta as taxas de DALY por mil habitantes, para cada grupo de causas de doenças, bem como sua distribuição proporcional. Evidencia-se que, no Brasil, os grupos com as maiores concentrações foram: IA (infecciosas e parasitárias - 9,2\%), IIE (doenças neuropsiquiátricas - 18,6\%), IIG (doenças cardiovasculares - $13,3 \%$ ) ellH (doenças respiratórias crônicas - 8,1\%). Este padrão é observado nas diferentes grandes regiões do País, ainda que com intensidades diferentes.

Grandes alterações podem ser visualizadas ao se comparar o ranking do DALY com os rankings dos seus componentes de morbidade (YLD) e mortalidade (YLL) (Tabela 4). Em apenas um único caso, o ranking da morbidade e da mortalidade coincidem (doenças infeccio- 


\section{Tabela 1}

Número absoluto e distribuição proporcional de YLL por grupos de causas, em ambos os sexos, segundo grandes regiões. Brasil, 1998.

\begin{tabular}{|c|c|c|c|c|c|c|c|c|c|c|c|c|}
\hline \multirow[t]{3}{*}{ Grupos de causas de óbito } & \multicolumn{12}{|c|}{ Grandes regiões } \\
\hline & \multicolumn{2}{|c|}{ Norte } & \multicolumn{2}{|c|}{ Nordeste } & \multicolumn{2}{|c|}{ Centro-Oeste } & \multicolumn{2}{|c|}{ Sudeste } & \multicolumn{2}{|c|}{ Sul } & \multicolumn{2}{|c|}{ Brasil } \\
\hline & YLL & $\%$ & YLL & $\%$ & YLL & $\%$ & YLL & $\%$ & YLL & $\%$ & YLL & $\%$ \\
\hline Todas as causas & 1.154 .090 & 100 & 6.081 .484 & 100 & 1.024 .519 & 100 & 7.413.882 & 100 & 2.357.296 & 100 & 18.031 .271 & 100 \\
\hline $\begin{array}{l}\text { Grupo I } \\
\text { Doenças infecciosas/ } \\
\text { parasitárias, maternas, } \\
\text { perinatais e nutricionais }\end{array}$ & 436.939 & 38 & 2.204 .001 & 35 & 259.927 & 25 & 1.609 .268 & 21 & 430.282 & 18 & 4.940 .416 & 26 \\
\hline I.A. Infecciosas e parasitárias & 135.175 & 12 & 793.050 & 13 & 97.988 & 10 & 584.749 & 8 & 149.027 & 6 & 1.759 .988 & 10 \\
\hline I.B. Infecções respiratórias & 63.275 & 5 & 344.547 & 6 & 41.954 & 4 & 384.394 & 5 & 98.581 & 4 & 932.7 & 5 \\
\hline I.C. Condições maternas & 7.736 & 1 & 31.479 & 1 & 4.262 & 0 & 27.357 & 0 & 9.684 & 0 & 80.519 & 0 \\
\hline $\begin{array}{l}\text { I.D. Condições durante } \\
\text { o período perinatal }\end{array}$ & 212.958 & 18 & 902.668 & 14 & 106.492 & 10 & 555.542 & 7 & 158.309 & 7 & 1.935 .970 & 10 \\
\hline I.E. Deficiências nutricionais & is 17.794 & 2 & 132.256 & 2 & 9.231 & 1 & 57.225 & 1 & 14.682 & 1 & 231.189 & 1 \\
\hline \multicolumn{13}{|l|}{ Grupo II } \\
\hline Doenças não-transmissíveis & s 554.100 & 48 & 3.288 .498 & 55 & 553.585 & 55 & 4.365 .587 & 60 & 1.549 .559 & 66 & 10.311 .329 & 59 \\
\hline II.A. Câncer & 118.122 & 10 & 587.363 & 10 & 113.083 & 11 & 965.559 & 13 & 385.294 & 16 & 2.169 .420 & 12 \\
\hline II.B. Neoplasias benignas & 1.911 & 0 & 7.857 & 0 & 2.743 & 0 & 8.395 & 0 & 3.478 & 0 & 24.384 & 0 \\
\hline II.C. Diabetes mellitus & 24.886 & 2 & 201.510 & 3 & 25.995 & 3 & 215.646 & 3 & 63.447 & 3 & 531.485 & 3 \\
\hline $\begin{array}{l}\text { II.D. D oenças en dócrinas } \\
\text { e metabólicas }\end{array}$ & 16.017 & 1 & 90.811 & 1 & 11.765 & 1 & 73.828 & 1 & 17.533 & 1 & 209.954 & 1 \\
\hline $\begin{array}{l}\text { II.E. Doenças } \\
\text { neuropsiquiátricas }\end{array}$ & 16.708 & 1 & 118.078 & 2 & 25.332 & 2 & 157.837 & 2 & 50.618 & 2 & 368.573 & 2 \\
\hline $\begin{array}{l}\text { II.F. D esordens de órgãos } \\
\text { do sentido }\end{array}$ & 126 & 0 & 708 & 0 & 104 & 0 & 245 & 0 & 74 & 0 & 1.256 & 0 \\
\hline $\begin{array}{l}\text { II.G. Doenças } \\
\text { cardiovasculares }\end{array}$ & 210.151 & 17 & 1.436 .924 & 24 & 235.029 & 22 & 1.938 .492 & 26 & 645.231 & 26 & 4.465 .827 & 24 \\
\hline $\begin{array}{l}\text { II.H. Doenças respiratórias } \\
\text { crônicas }\end{array}$ & 45.756 & 4 & 252.355 & 4 & 44.744 & 4 & 336.160 & 5 & 163.072 & 7 & 842.088 & 5 \\
\hline $\begin{array}{l}\text { II.I. Doenças do aparelho } \\
\text { digestivo }\end{array}$ & 70.028 & 6 & 406.345 & 7 & 57.956 & 6 & 515.294 & 7 & 158.244 & 7 & 1.207 .866 & 7 \\
\hline II.J. Doenças gênito-urinárias & as 17.903 & 2 & & 1 & & 1 & & 1 & & 1 & & 1 \\
\hline II.K. Doenças de pele & 2.156 & 0 & 11.488 & 0 & 1.281 & 0 & 12.277 & 0 & 2.542 & 0 & 29.743 & 0 \\
\hline $\begin{array}{l}\text { II.L. Doenças } \\
\text { músculo-esqueléticas }\end{array}$ & 3.930 & 0 & 18.010 & 0 & 3.212 & 0 & 18.131 & 0 & 7.117 & 0 & 50.399 & 0 \\
\hline II.M. Anomalias congênitas & 39.801 & 3 & 155.579 & 3 & 30.862 & 3 & 140.194 & 2 & 64.603 & 3 & & 2 \\
\hline II.N. Condições orais & 253 & 0 & 966 & 0 & 27 & 0 & 352 & 0 & 152 & 0 & 1.749 & 0 \\
\hline \multicolumn{13}{|l|}{ Grupo III } \\
\hline Causas externas & 163.051 & 14 & 588.985 & 10 & 211.007 & 20 & 1.439 .027 & 19 & 377.455 & 16 & 2.779 .526 & 15 \\
\hline $\begin{array}{l}\text { III.A. Causas externas } \\
\text { não-intencionais }\end{array}$ & 88.708 & 8 & 309.531 & 5 & 114.725 & 11 & 647.705 & 9 & 232.529 & 10 & 1.393 .198 & 8 \\
\hline $\begin{array}{l}\text { III.B. Causas externas } \\
\text { intencionais }\end{array}$ & 74.343 & 6 & 279.454 & 5 & 96.283 & 9 & 791.321 & 10 & 144.927 & 6 & 1.386 .328 & 7 \\
\hline
\end{tabular}

Fonte: Projeto Carga de Doença. Brasil, 1998. EN SP/Fiocruz. 
Tabela 2

YLD por 1.000 habitantes e sua distribuição proporcional por grupos de causas, em ambos os sexos, segundo grandes regiões. Brasil, 1998.

\begin{tabular}{|c|c|c|c|c|c|c|c|c|c|c|c|c|}
\hline \multirow[t]{3}{*}{ Grupos de causas de óbito } & \multicolumn{12}{|c|}{ Grandes regiões } \\
\hline & \multicolumn{2}{|c|}{ Norte } & \multicolumn{2}{|c|}{ Nordeste } & \multicolumn{2}{|c|}{ Centro-Oeste } & \multicolumn{2}{|c|}{ Sudeste } & \multicolumn{2}{|c|}{ Sul } & \multicolumn{2}{|c|}{ Brasil } \\
\hline & Taxa & $\%$ & Taxa & $\%$ & Taxa & $\%$ & Taxa & $\%$ & Taxa & $\%$ & Taxa & $\%$ \\
\hline Todas as causas & 112 & 100 & 117 & 100 & 108 & 100 & 129 & 100 & 113 & 100 & 120 & 100 \\
\hline \multicolumn{13}{|l|}{ Grupo I } \\
\hline $\begin{array}{l}\text { Doenças infecciosas/parasitárias, } \\
\text { maternas, perinatais e nutricionais }\end{array}$ & 29 & 26,3 & 27 & 23 & 22 & 20,5 & 22 & 17,2 & 21 & 18,7 & 24 & 19,8 \\
\hline I.A. Infecciosas e parasitárias & 16 & 14,1 & 14 & 12,1 & 9 & 7,9 & 8 & 6,3 & 8 & 6,9 & 10 & 8,6 \\
\hline I.B. Infecções respiratórias & 3 & 2,3 & 2 & 2,1 & 2 & 2,2 & 2 & 1,7 & 2 & 2 & 2 & 1,9 \\
\hline I.C. Condições maternas & 4 & 3,3 & 4 & 3,3 & 5 & 4,4 & 6 & 4,7 & 5 & 4,7 & 5 & 4,2 \\
\hline I.D. Condições do período perinatal & 3 & 3 & 3 & 2,5 & 3 & 2,6 & 3 & 2 & 3 & 2,3 & 3 & 2,3 \\
\hline I.E. Deficiências nutricionais & 4 & 3,5 & 4 & 3,2 & 4 & 3,4 & 3 & 2,4 & 3 & 2,8 & 3 & 2,8 \\
\hline \multicolumn{13}{|l|}{ Grupo II } \\
\hline Doenças não-transmissíveis & 77 & 68,6 & 84 & 71,8 & 79 & 73,5 & 100 & 77,6 & 84 & 74,5 & 90 & 74,7 \\
\hline II.A. Câncer & 1 & 0,5 & 1 & 0,8 & 1 & 0,8 & 2 & 1,3 & 1 & 1,1 & 1 & 1 \\
\hline II.B. Neoplasias benignas & - & - & - & - & - & - & - & - & - & - & - & - \\
\hline II.C. Diabetes mellitus & 7 & 6,2 & 6 & 5,1 & 6 & 5,5 & 11 & 8,3 & 10 & 8,8 & 9 & 7,2 \\
\hline II.D. Doenças endócrinas e metabólicas & 2 & 2,1 & 3 & 2,9 & 2 & 1,8 & 2 & 1,4 & 1 & 1,1 & 2 & 1,8 \\
\hline II.E. Doenças neuropsiquiátricas & 38 & 33,8 & 39 & 32,9 & 40 & 37,1 & 43 & 33,6 & 41 & 36,1 & 41 & 34 \\
\hline II.F. Desordens de órgãos do sentido & 2 & 1,5 & 2 & 2 & 2 & 1,8 & 3 & 2,1 & 3 & 2,3 & 2 & 2 \\
\hline II.G. D oenças cardiovasculares & 2 & 2,2 & 3 & 2,6 & 3 & 2,7 & 3 & 2,7 & 3 & 3,1 & 3 & 2,7 \\
\hline II.H. D oenças respiratórias crônicas & 13 & 11,8 & 15 & 12,9 & 14 & 12,9 & 13 & 9,8 & 13 & 11,6 & 14 & 11,2 \\
\hline II.I. D oenças do aparelho digestivo & 0 & 0,3 & 0 & 0,4 & 0 & 0,4 & 0 & 0,2 & 1 & 0,6 & 0 & 0,4 \\
\hline II.J. D oenças gênito-urinárias & 0 & 0,2 & 0 & 0 & 0 & 0,2 & 1 & 0,5 & 1 & 0,5 & 0 & 0,3 \\
\hline II.K. Doenças de pele & - & - & - & - & - & - & - & - & - & - & - & - \\
\hline II.L. D oenças músculo-esqueléticas & 6 & 5,6 & 10 & 8,4 & 7 & 6,2 & 18 & 14,3 & 6 & 5,6 & 12 & 10,4 \\
\hline II.M. Anomalias congênitas & 2 & 1,5 & 2 & 1,5 & 2 & 1,4 & 2 & 1,2 & 1 & 1,3 & 2 & 1,3 \\
\hline II.N. Condições orais & 3 & 2,8 & 3 & 2,3 & 3 & 2,6 & 3 & 2,3 & 3 & 2,5 & 3 & 2,4 \\
\hline \multicolumn{13}{|l|}{ Grupo III } \\
\hline Causas externas & 6 & 5,1 & 6 & 5,2 & 6 & 6 & 7 & 5,2 & 8 & 6,8 & 7 & 5,5 \\
\hline III .A. Causas externas não-intencionais & 5 & 4,6 & 6 & 4,9 & 6 & 5,8 & 6 & 4,8 & 7 & 6,6 & 6 & 5,1 \\
\hline III.B. Causas externas intencionais & 1 & 0,5 & 0 & 0,3 & 0 & 0,2 & 1 & 0,4 & 0 & 0,2 & 0 & 0,3 \\
\hline
\end{tabular}

Fonte: Projeto Carga de Doença. Brasil, 1998. ENSP/Fiocruz. 
DALY por 1.000 habitantes e sua distribuição proporcional por grupos de causas, em ambos os sexos, segundo grandes regiões. Brasil, 1998.

\begin{tabular}{|c|c|c|c|c|c|c|c|c|c|c|c|c|}
\hline \multirow[t]{3}{*}{ Grupos de causas de óbito } & \multicolumn{12}{|c|}{ Grandes regiões } \\
\hline & \multicolumn{2}{|c|}{ Norte } & \multicolumn{2}{|c|}{ Nordeste } & \multicolumn{2}{|c|}{ Centro-0 este } & \multicolumn{2}{|c|}{ Sudeste } & \multicolumn{2}{|c|}{ Sul } & \multicolumn{2}{|c|}{ Brasil } \\
\hline & Taxa & $\%$ & Taxa & $\%$ & Taxa & $\%$ & Taxa & $\%$ & Taxa & $\%$ & Taxa & $\%$ \\
\hline Todas as causas & 209 & 100 & 250 & 100 & 201 & 100 & 236 & 100 & 210 & 100 & 232 & 100 \\
\hline $\begin{array}{l}\text { Grupo I } \\
\text { Doenças infecciosas/ } \\
\text { parasitárias, maternas, perinatais } \\
\text { e nutricionais }\end{array}$ & 66 & 32,7 & 75 & 30,1 & 46 & 22,8 & 45 & 19,2 & 39 & 18,5 & 54 & 23,5 \\
\hline I.A. Infecciosas e parasitárias & 27 & 13 & 31 & 12,6 & 17 & 8,7 & 17 & 7 & 14 & 6,6 & 21 & 9,2 \\
\hline I.B. Infecções respiratórias & 8 & 3,8 & 10 & 4 & 6 & 3,1 & 8 & 3,3 & 6 & 3 & 8 & 3,5 \\
\hline I.C. Condições maternas & 4 & 2,1 & 4 & 1,8 & 5 & 2,5 & 6 & 2,7 & 6 & 2,7 & 6 & 2,4 \\
\hline I.D. Condições do período perinatal & 21 & 10,2 & 23 & 9 & 12 & 6,2 & 11 & 4,5 & 9 & 4,3 & 15 & 6,4 \\
\hline I.E. Deficiências nutricionais & 5 & 2,6 & 7 & 2,6 & 5 & 2,3 & 4 & 1,7 & 4 & 1,8 & 5 & 2,1 \\
\hline \multicolumn{13}{|l|}{ Grupo II } \\
\hline Doenças não-transmissíveis & 124 & 59 & 156 & 62,4 & 129 & 64,5 & 163 & 69,1 & 148 & 70,4 & 154 & 66,3 \\
\hline II.A. Câncer & 11 & 5 & 14 & 5,5 & 11 & 5,6 & 16 & 6,6 & 17 & 8,2 & 15 & 6,3 \\
\hline II.B. Neoplasias benignas & 0 & 0,1 & 0 & 0,1 & 0 & 0,1 & 0 & 0,1 & 0 & 0,1 & 0 & 0,1 \\
\hline II.C. Diabetes mellitus & 9 & 4,3 & 10 & 4,1 & 8 & 4,1 & 14 & 5,9 & 13 & 6 & 12 & 5,1 \\
\hline II.D. D oenças endócrinas e metabólicas & 4 & 1,8 & 5 & 2,1 & 3 & 1,5 & 3 & 1,2 & 2 & 0,9 & 4 & 1,5 \\
\hline II.E. D oenças neuropsiquiátricas & 39 & 18,8 & 41 & 16,5 & 42 & 21 & 46 & 19,3 & 43 & 20,3 & 43 & 18,6 \\
\hline II.F. D esordens de órgãos do sentido & 2 & 0,8 & 2 & 0,9 & 2 & 1 & 3 & 1,1 & 3 & 1,2 & 2 & 1,1 \\
\hline II.G. Doenças cardiovasculares & 20 & 9,6 & 34 & 13,8 & 24 & 12,1 & 32 & 13,4 & 30 & 14,4 & 31 & 13,3 \\
\hline II.H. Doenças respiratórias crônicas & 17 & 8,2 & 21 & 8,3 & 18 & 9 & 18 & 7,4 & 20 & 9,4 & 19 & 8,1 \\
\hline II.I. Doenças do aparelho digestivo & 5 & 2,5 & 7 & 3 & 5 & 2,3 & 6 & 2,6 & 6 & 2,7 & 6 & 2,7 \\
\hline II.J. Doenças gênito-urinárias & 2 & 0,8 & 2 & 0,8 & 2 & 0,8 & 2 & 0,9 & 2 & 0,8 & 2 & 0,8 \\
\hline II.K. Doenças de pele & 0 & 0,1 & 0 & 0,1 & 0 & 0,1 & 0 & 0,1 & 0 & 0,1 & 0 & 0,1 \\
\hline II.L. Doenças músculo-esqueléticas & 7 & 3,1 & 10 & 4,1 & 7 & 3,5 & 19 & 7,9 & 7 & 3,1 & 13 & 5,5 \\
\hline II.M. Anomalias congênitas & 5 & 2,4 & 5 & 2 & 4 & 2,2 & 4 & 1,5 & 4 & 1,9 & 4 & 1,8 \\
\hline II.N. Condições orais & 3 & 1,5 & 3 & 1,1 & 3 & 1,4 & 3 & 1,2 & 3 & 1,3 & 3 & 1,2 \\
\hline \multicolumn{13}{|l|}{ Grupo III } \\
\hline Causas externas & 19 & 9,3 & 19 & 7,6 & 26 & 12,8 & 28 & 11,7 & 23 & 11,1 & 24 & 10,2 \\
\hline III.A. Causas externas não-intencionais & 13 & 6 & 12 & 5 & 17 & 8,3 & 16 & 6,6 & 17 & 8,1 & 15 & 6,4 \\
\hline III.B. Causas externas intencionais & 7 & 3,3 & 6 & 2,6 & 9 & 4,5 & 12 & 5,1 & 6 & 3 & 9 & 3,9 \\
\hline
\end{tabular}

Fonte: Projeto Carga de Doença. Brasil, 1998. EN SP/Fiocruz. 
Tabela 4

Número de DALY, YLD, YLL, óbitos e rankings correspondentes para grupos de causas. Brasil, 1998.

\begin{tabular}{|c|c|c|c|c|c|c|c|c|}
\hline Grupos de causas de óbito & DALY & Posto & YLD & Posto & YLL & Posto & Óbitos & Posto \\
\hline Todas as causas & 37.518 .239 & - & 19.486 .968 & - & 18.031.271 & - & 1.129 .843 & - \\
\hline $\begin{array}{l}\text { Grupo I } \\
\text { Doenças infecciosas/parasitárias, } \\
\text { maternas, perinatais e nutricionais }\end{array}$ & 8.805 .213 & - & 3.864 .796 & - & 4.940 .416 & - & 224.367 & - \\
\hline I.A. Infecciosas e parasitárias & 3.438 .619 & 3 & 1.678 .631 & 4 & 1.759 .988 & 4 & 83.791 & 3 \\
\hline I.B. Infecções respiratórias & 1.310 .824 & 11 & 378.073 & 13 & 932.751 & 8 & 61.059 & 7 \\
\hline I.C. Condições maternas & 891.278 & 13 & 810.758 & 7 & 80.519 & 16 & 3.112 & 17 \\
\hline $\begin{array}{l}\text { I.D. Condições durante } \\
\text { o período perinatal }\end{array}$ & 2.383 .012 & 6 & 447.042 & 11 & 1.935 .970 & 3 & 63.974 & 5 \\
\hline I.E. D eficiências nutricionais & 781.480 & 14 & 550.292 & 8 & 231.189 & 14 & 12.431 & 15 \\
\hline $\begin{array}{l}\text { Grupo II } \\
\text { Doenças não-transmissíveis }\end{array}$ & 24.867 .484 & - & 14.556.156 & - & 10.311 .329 & - & 787.876 & - \\
\hline II.A. Câncer & 2.370 .331 & 7 & 200.910 & 16 & 2.169 .420 & 2 & 153.449 & 2 \\
\hline II.B. Neoplasias benignas & 24.384 & 21 & - & 20 & 24.384 & 19 & 1.543 & 19 \\
\hline II.C. Diabetes mellitus & 1.929 .362 & 9 & 1.397 .878 & 5 & 531.485 & 10 & 43.811 & 10 \\
\hline II.D. Doenças endócrinas e metabólicas & 567.046 & 16 & 357.092 & 14 & 209.954 & 15 & 13.788 & 14 \\
\hline II.E. Doenças neuropsiquiátricas & 6.987 .074 & 1 & 6.618 .501 & 1 & 368.573 & 12 & 20.047 & 11 \\
\hline II.F. Desordens de órgãos do sentido & 395.967 & 18 & 394.711 & 12 & 1.256 & 21 & 81 & 21 \\
\hline II.G. Doenças cardiovasculares & 4.989 .406 & 2 & 523.579 & 9 & 4.465 .827 & 1 & 381.202 & 1 \\
\hline II.H. D oenças respiratórias crônicas & 3.033 .382 & 4 & 2.191 .295 & 2 & 842.088 & 9 & 74.559 & 4 \\
\hline II.I. D oenças do aparelho digestivo & 1.001 .374 & 12 & 68.441 & 17 & 932.933 & 7 & 59.886 & 8 \\
\hline II.J. D oenças genito-urinárias & 316.040 & 19 & 63.560 & 19 & 252.480 & 13 & 19.305 & 12 \\
\hline II.K. Doenças de pele & 29.743 & 20 & - & 20 & 29.743 & 18 & 2.247 & 18 \\
\hline II.L. D oenças músculo-esqueléticas & 2.072 .591 & 8 & 2.022 .192 & 3 & 50.399 & 17 & 3.168 & 16 \\
\hline II.M. Anomalias congênitas & 687.153 & 15 & 256.116 & 15 & 431.038 & 11 & 14.702 & 13 \\
\hline II.N. Condições orais & 463.630 & 17 & 461.881 & 10 & 1.749 & 20 & 89 & 20 \\
\hline \multicolumn{9}{|l|}{ Grupo III } \\
\hline Causas externas & 3.845 .541 & - & 1.066 .016 & - & 2.779 .526 & - & 117.600 & - \\
\hline III.A. Causas externas não-intencionais & 2.393 .284 & 5 & 1.000 .086 & 6 & 1.393 .198 & 5 & 61.748 & 6 \\
\hline III.B. Causas externas intencionais & 1.452 .257 & 10 & 65.929 & 18 & 1.386 .328 & 6 & 55.852 & 9 \\
\hline
\end{tabular}

Fonte: Projeto Carga de Doença. Brasil, 1998. EN SP/Fiocruz.

sas e parasitárias). No ranking do DALY, as doenças do grupo II.E (neuropsiquiátricas) ocupam a primeira posição. As doenças desse grupo haviam sido classificadas em 12a posição em termos de mortalidade (YLL), portanto, bastante diferente do seu ranking final, quando se utiliza a quantidade de DALY cal culado. 0 subgrupo das doenças músculo-esqueléticas encontravam-se na 17ạ posição em termos de YLL e passaram para a 3a e 8a posições no cômputo deYLD e DALY respectivamente. As neoplasias (II.A) têm um peso maior na componente mortalidade ( 2 a posição) do que na morbidade (16ạ posição).

A segunda posição no ranking final, obtido através do DALY, foi ocupada pelas doenças cardiovasculares (grupo II.G). Esse agrupa- mento havia ficado na 1a posição, tanto em número de óbitos, quanto em quantidade de YLL; mas em termos de YLD, esse grupo ocupou a 9a posição.

O diabetes mellitus, grupo II.C, ocupou a nona posição no ranking final do DALY, 5 a e $10 a$ posição em termos deYLL eYLD respectivamente. As causas externas intencionais ficaram em 10a posição no ranking do DALY, bem no meio entre a $18^{a}$ posição, na morbidade, e $6^{a}$ posição, na mortalidade.

Analisando o ranking das principais causas de anos de vida perdidos por morte prematura ou por incapacidade (DALY) para Brasil e macrorregiões (dados não apresentados), evidenciou-se que, para o Brasil como um todo, 0 diabetes mellitus $(5,1 \%)$, as doenças isquêmi- 
cas do coração $(5,0 \%)$ e as doenças cerebrovasculares - AVC - primeiro ataque $(4,6 \%)$ englobaram $14,7 \%$ do total do DALY e são características de um padrão epidemiológico de países desenvolvidos. Em contraposição, observa-se que o País ainda apresenta condições que refletem a pobreza e precariedade no atendimento e prevenção à saúde, como a asfixia e traumatismo ao nascer (3,8\%). Chamamos a atenção para a ocorrência da presença do transtorno depressivo recorrente (episódio de depressão) como quarta causa de DALY (3,8\%). As doenças mentais englobam 10,8\% do total de DALY em ambos os sexos.

A cirrose hepática com 1,5\% do total Brasil e 2,2\% no sexo masculino está entre as vinte primeiras causas em ambos os sexos, em todas as regiões, exceto na região Norte. No sexo masculino, encontra-se entre as vinte primeiras causas em todas as regiões, com valores entre $2,4 \%$ e $1,7 \%$.

Apresentamos na tabela 5 a distribuição proporcional, por região e sexo, das principais causas de DALY agrupadas de acordo com as seguintes categorias: causas externas, mentais, crônicas cardiovasculares (diabetes, doença is- quêmica do coração - IAM e angina e doenças cerebrovasculares - infarto cerebral e primeiro episódio de AVC), crônicas pulmonares (DPOC, asma e infecções de vias aéreas inferiores) e doenças decorrentes da pobreza e/ou de precárias condições de acesso/atendimento à saúde (asfixia e traumatismo ao nascer, episódios de diarréia, anemia por deficiência de ferro, aborto, septicemia do recém-nascido, baixo peso ao nascer, desnutrição protéico-calórica, tuberculose, doença de Chagas e tracoma).

Destaca-se nessa tabela a transição epidemiológica que o País atravessa, assim como os diferenciais regionais nas condições de atenção à saúde e de desenvolvimento econômico. Verificamos que as doen ças decorrentes de causas externas ocorrem mais entre os homens e nas regiões Sudeste, Centro-O este e Sul; as doenças mentais tradicionalmente não aparecem como uma causa relevante nas estatísticas de saúde, já que assumem valores baixos de mortalidade, mas possuem, todavia, um grande peso de incapacidade e duração longa. Contribuem com um percentual expressivo de DALY igualmente em todas as regiões, principalmente, entre as mulheres; as doenças crônicas cardiovasculares

\section{Tabela 5}

Distribuição proporcional dos cinco principais grupos de causas, considerando os DALYs, segundo sexo e grandes regiões. Brasil, 1998.

\begin{tabular}{|c|c|c|c|c|c|}
\hline \multirow[t]{2}{*}{ Região } & \multicolumn{5}{|c|}{ Causas } \\
\hline & Externas & Mentais & $\begin{array}{c}\text { Crônicas } \\
\text { Cardiovasculares }\end{array}$ & $\begin{array}{c}\text { Crônicas } \\
\text { Pulmonares }\end{array}$ & $\begin{array}{c}\text { Relacionadas à } \\
\text { "pobreza/ acesso } \\
\text { precário atendimento" }\end{array}$ \\
\hline Brasil & 7,5 & 10,8 & 14,7 & 8,1 & 8,6 \\
\hline Masculino & 12,9 & 10,4 & 14,6 & 8,3 & 6,1 \\
\hline Feminino & 2,2 & 12,7 & 14,9 & 7,7 & 11,3 \\
\hline Norte & 6,9 & 10,6 & 11,5 & 8,4 & 15,6 \\
\hline M asculino & 10,2 & 10,8 & 12 & 8,8 & 13,3 \\
\hline Feminino & 1,3 & 11,7 & 11,1 & 8,3 & 18,8 \\
\hline Nordeste & 5,5 & 9,5 & 13,9 & 8,1 & 13,8 \\
\hline Masculino & 8,6 & 8,6 & 13,7 & 8,2 & 14,2 \\
\hline Feminino & 1 & 10,2 & 14,2 & 7,9 & 17,5 \\
\hline Centro-0 este & 9,7 & 11,9 & 12,7 & 8 & 8,8 \\
\hline Masculino & 14,3 & 11,9 & 12,9 & 8 & 7 \\
\hline Feminino & 3,3 & 14,8 & 12,5 & 7,9 & 12,2 \\
\hline Sudeste & 10,2 & 11,6 & 15,5 & 7,7 & 5,3 \\
\hline Masculino & 15,4 & 10,4 & 15,2 & 8 & 2,7 \\
\hline Feminino & 1,4 & 14,3 & 15,6 & 7,4 & 8,3 \\
\hline Sul & 8,5 & 11,8 & 17 & 8,9 & 5,5 \\
\hline Masculino & 13,7 & 11 & 16,7 & 9,4 & 2,7 \\
\hline Feminino & 2,7 & 14,2 & 17,3 & 8,2 & 8,4 \\
\hline
\end{tabular}

Fonte: Projeto Carga de Doença. Brasil, 1998. EN SP/Fiocruz. 
têm uma distribuição semelhante entre os sexos e maior expressão nas regiões Sul e Sudeste; as doenças crônicas pulmonares apresentam discreto aumento percentual entre os homens e maior ocorrência na região Sul; e, finalmente, as doenças relacionadas à pobreza e/ou precárias condições de atendimento à saúde que acometem principalmente as mulheres e as regiões Norte e Nordeste.

\section{D iscussão e considerações finais}

Este estudo nos dá um panorama da transição epidemiológica pela qual passa o Brasil. Segundo os resultados apresentados, as doenças crônico-degenerativas responderam por $66,3 \%$ da carga de doença no Brasil; as doenças infecciosas responderam por $23,5 \%$; e as causas externas foram responsáveis por $10,2 \%$.

Mundial mente, a carga de doença relacionada aos agravos não-transmissíveis tem se elevado rapidamente e sua prevenção tem sido 0 maior desafio para a saúde pública. A inatividade física juntamente com as dietas chamadas "não saudáveis" têm elevado a incidência das doenças não-transmissíveis, incluindo as doenças cardiovasculares, diabetes tipo 2 e certos tipos de câncer, explicando assim a importância destas doen ças nas estimativas da carga global de doença (WHO, 2003).

A utilização do indicador DALY neste trabalho mostra-se como uma iniciativa inovadora ao acoplar dois componentes: morbidade e mortalidade. A inclusão, no indicador, da perda de vida em função da incapacidade é relevante pelo menos em dois aspectos: primeiro, porque traz ao debate as conseqüências não fatais da doença, tão pouco medidas e utilizadas como indicadores de necessidades de saúde (M urray \& Lopez, 1996a); e segundo, porque facilita as decisões sobre a alocação de recursos, dando melhores subsídios aos gestores de saúde.

Estudos da carga de doença, por meio de seu indicador DALY, se colocam de forma decisiva na contribuiç̧ão para caracterizar o perfil de saúde da população no Brasil, já que, ao abordar conjuntamente as incapacidades, refletem de modo mais adequado o impacto da doença sobre as famílias, o sistema de saúde e, principalmente, a qualidade de vida (Ramos et al., 1993). É notável esta afirmativa quando se observa a grande carga de incapacidade gerada pelo subgrupo das doenças neuropsiquiátricas (IIE).
Ao analisarmos os anos de vida perdidos por morte prematura (YLL), verifica-se que as "doenças da modernidade" se destacam. No entanto, chama a atenção a posição ocupada pelo subgrupo das doenças cardiovasculares (1a posição), apresentando expressiva mortalidade que certamente deve ser alvo do uso deficiente das tecnologias disponíveis e da falta de acesso. Já em relação ao câncer, os tipos que afetam a traquéia/brônquios/pulmões e estômago foram os mais importantes para o Brasil e macrorregiões. Modos deficientes de industrialização, urbanização, consumo de substâncias nocivas como o tabaco, além de mudanças expressivas no estilo de vida da população brasileira podem ser, em parte, considerados fatores importantes para os resultados encontrados.

0 aumento nas taxas de mortalidade e morbidade por doenças cardiovasculares (DCV) vem ocorrendo nos últimos anos nos países em desenvolvimento (Avezum et al., 1999). Essas doenças são as que mais contribuem para a mortalidade e morbidade precoces, sendo responsáveis por 85 milhões de anos de vida perdidos ajustados pela incapacidade - DALY, soma dos anos de vida perdidos devido à morte prematura e os anos vividos com incapacidade ajustados segundo a severidade (Murray \& Lopez, 1996).

Projeções para 0 ano de 2020 indicam que as DCV permanecerão como a principal causa de mortalidade e incapacidade e que os DALYs atribuíveis às DCV irão se el evar para 140-160 milhões, sendo que a maior parte deste incremento ocorrerá nos países em desenvolvimento (Murray \& Lopez, 1996).

Quando analisamos a soma dos dois componentes (DALY) para o Brasil observa-se que o grupo I (doenças infecciosas parasitárias, maternas, perinatais e nutricionais) juntamente com grupo III (causas externas) contribuíram com percentuais expressivos em relação aos anos de vida perdidos por morte precoce e incapacidade. Diferenças importantes são verificadas quando se analisam as regiões, mostrando que as doenças do grupo I ainda são marcantes no Norte e Nordeste e as causas externas (grupo III) com as maiores proporções na região Centro-O este, Sudeste e Sul. Neste último grupo, a violência (agressões/negligência/abandono) e os acidentes de trânsito (traumatismos em colisão) apresentaram-se com perda importante de DALY no Brasil e grandes regiões. Este cenário demonstra as desigualdades além dos diferenciais nas mani- 
festações dos agravos segundo grupos sociais e regiões. Apontam para o modelo polarizado prolongado (justaposi ção das doenças infecciosas e parasitárias com as crônico-degenerativas) descrito por Frenk et al., (1991). Estes resultados reforçam a importância de se considerar a morbidade um aspecto relevante na gestão e financiamento do Sistema Único de Saúde.

Projeções da OMS para 2025 incluem o Brasil entre os dez países do mundo com maior contingente de pessoas com 60 anos e mais. A proporção de idosos no Brasil passou de 6,3\% em 1980 para 7,6\% em 1996, estimando-se $14 \%$ em 2025. Esta mudança demográfica corresponde a uma transição epidemiológica que resulta em um importante crescimento da demanda aos serviços sociais e de saúde ( Garcia et al., 2002). Os agravos crônico-degenerativos, que atingem esta faixa etária, implicam tratamento de duração mais longa e recuperação mais lenta e complicada, exigindo também intervenções de el evado custo.

O cenário projetado para 2020 por Murray \& Lopez (1996) para o indicador DALY evidencia que entre as cinco principais causas de anos de vida perdidos por morte precoce e incapacidade estão: as doenças isquêmicas do coração, depressão, acidentes de trânsito, doenças cerebrovasculares e doenças pulmonares obstrutivas crônicas. A importância das doenças crônico-degenerativas como problema de saúde pública requer a reestruturação do sistema nos níveis básico e hospitalar no sentido de promover a prevenção, diagnóstico, tratamento precoce das incapaci dades geradas por estes agravos (Ramos et al., 1993).

Os custos crescentes para os sistemas de saúde em relação ao impacto das complicações relacionadas aos agravos não-transmissíveis merecem destaque. Aproximadamente $40 \%$ $60 \%$ das amputações não traumáticas em membros inferiores são realizadas em pacientes com diabetes (Grupo de Trabalho Internacional sobre Pé Diabético, 2001). O utra complicação freqüente associada a diabetes tem sido a Insuficiência Renal Crônica, IRC (Bruno \& Gross, 2000; Klag et al., 1996). Estas complicações representam um problema econômico significativo para o País.

As doenças cardiovasculares e seus fatores de risco, as neoplasias, as doenças respiratórias crônicas, as doenças osteo-músculo-articulares, os acidentes automobilísticos e do trabalho e as conseqüências da violência social têm um papel decisivo nos dispêndios da saúde, da previdência e nos gastos, além deinfluenciarem na qualidade de vida das pessoas acometidas e de seus familiares. Se somarmos a perda de mãode-obra qualificada e de difícil reposição, os anos de vida produtiva e horas de trabal ho perdidos, os gastos com aposentadorias precoces por doenças e com a utilização crescente e acrítica ou até abusiva de procedimentos diagnósticos e terapêuticos de alta tecnologia, observaremos que a não adoção de mecanismos adequados de prevenção das enfermidades nãotransmissíveis comprometerá o desenvolvimento do País. Quando implementadas com novos modelos de inter venção que considerem o contexto social e o meio ambiente no qual se vive, as estratégias de promoção e prevenção à saúde podem reduzir significativamente a morbi-mortalidade pelas enfermidades não-transmissíveis (Silva \& M aldonato, 2003).

O uso racional dos recursos disponíveis torna imprescindível em saúde pública a busca permanente de instrumentos que ajudem a tomada de decisões. Os indicadores de saúde cumprem esse papel e suas características determinam a extensão de seu uso (Nedel et al., 1999). A utilização do indicador DALY propicia a identificação de prioridades em função do perfil epidemiológico, facilitando a tomada de decisões sobre a destinação de recursos, além de torná-las mais explícitas à sociedade. Permite também o estabelecimento de prioridades de investigação em saúde, a determinação de iniqüidade (ou desigualdades) para tomada de decisões com o objetivo de superar as diferenças encontradas (M urray et al., 1995; Fleury, 1995; Lozano et al., 1995).

\section{Colaboradores}

M A Schramm participou de todas as etapas do estudo "Carga de doença no Brasil" e da elaboração final do artigo; AF Oliveira, da elaboração da introdução do estudo e discussão dos resultados; IC Leite e JG Valente participaram de todas as etapas do estudo "Carga de doença no Brasil" e da revisão final do artigo; AMJ Gadelha, M C Portela e M R Campos participaram de todas as etapas do estudo "Carga de doença no Brasil". 


\section{Referências bibliográficas}

Azevum A, Neto JM R \& Piegas L 1999. Why do we need randomized and epidemiological studies on cardiovascular disease? Evidence-based cardiology VII. Arquivo Brasileiro de Cardiologia 72(3):289-295.

Arnand S \& Hanson K 1997. Disability-Adjusted Life of Years: a critical review. Journal of $\mathrm{H}$ ealth Economics 16:685-702

Barreto M L \& Carmo E 1995. Mudanças em padrões de morbi-mortalidade: conceitos e métodos, pp. 17-30. In C M onteiro (org.). Velhos e novos males da saúde no Brasil. Hucitec, São Paulo.

Barreto M L, Carmo E, Noronha CV, Neves BRT \& Alves PC 1993. Mudança nos padrões de morbi-mortalidade: uma revisão crítica das abordagens epidemiológicas. Physis 3:126-146.

Bobadilla JL 1996. Priority setting and cost effectiveness, pp. 43-60. In Janovsky K (ed). Health Policy Systems Development: An Agenda for Research. Geneva: WHO.

Bruno RM \& Gross JL 2000. Prognostic factors in Brazilian diabetic patients starting dialysis - A 3,6-year follow-up study. Journal of Diabetes and its Complications 14:266-271.

Chaimowicz F 1997. A saúde dos idosos brasileiros às vésperas do século 21: problemas, projeções e alternativas. Revista de Saúde Pública 31(2):184-200.

Coale A \& Guo G 1989. Revised regional model life tables at very low levels of mortality. Population Index 55 (4):613-643.

Dasgupta P, Sem A \& M arglin S 1972. Guidelines for project evaluation. United Nations, Nova York.

Fleury S 1995. Iniqüidades nas políticas de saúde: o caso da América Latina. Revista de Saúde Pública 29:243-250.

Frenk J et al. 1991. La transición epidemiológica en Amé rica Latina. Boletín de la O ficina Sanitaria Panamericana 111(6):485-496.

Gadelha AMJ et al. 2002. Relatório Final do Projeto Estimativa da Carga de Doença do Brasil - 1998. ENSP/ Fiocruz-FEN SPTEC, Rio de Janeiro.

Garcia M AA, Rodrigues M G \& Borega RS 2002. 0 enveIhecimento e a saúde. Revista de Ciências M édicas 11(3):221-231.

Goulart FAA 1999. Cenários epidemiológicos, demográficos e institucionais para os modelos de atenção à saúde. Informe Epidemiológico do SU S 8(2):17-26.

Gold M R, Siegel JE, Weinstein MC \& Russell LB 1996. Cost-effectiveness in $\mathrm{H}$ ealth and Medicine. Oxford University Press, Nova York.

Grupo de Trabalho Internacional sobre PéDiabético 2001. Consenso Internacional sobre Pé Diabético. Secretaria de Estado de Saúde do Distrito Federal, Brasília.

Klag MJ et al. 1996. Blood pressure and end-stage renal disease in men. New England Journal of Medicine 334:13-18.

Layard R \& Gleister S 1994. Cost-benefit analysis. Cambridge University Press, Cambridge.

Lerner M 1973. M odernization and health: a model of the health transition. Documento apresentado na Reunião Anual da American Public Health Association, São Francisco, Califórnia (inédito).

Lind RC, Stiglitz JE, Wilson R, Dasgupta P \& Stockfisch JA 1982. Discounting for timeand risk in energy policy. Johns Hopkins University Press, Baltimore.
Lozano $R$ et al. 1995. Burden of disease assessment and health system reform: results of a study in M éxico. Journal of International Development 7:555-563.

Murray CJL \& Lopez A 1996. The Global Burden of Disease: a comprehensive assessment of mortality and disability from diseases, injuries, and risk factors in 1990 and projected to 2020. Harvard School of Public $\mathrm{H}$ ealth, Harvard.

Murray CJL \& Lopez AD 1996a. Estimating causes of death: new methods and global and regional applications for 1990, pp. 117-200. In CJL Murray \& AD Lopez (ed). The Global Burden of Disease: a comprehensive assessment of mortality and disability from diseases, injuries, and risk factors in 1990 and projected to 2020. Harvard School of Public Health, Harvard.

Murray CJL \& Lopez A 1996b. The Global Burden of Disease in 1990: final results and their sensitivity to alternative epidemiologic perspectives, discount rates, age-weightsand disability weights, pp. 246-293. In CJL Murray \& AD Lopez (ed). The Global Burden of $D$ isease: a comprehensive assessment of mortality and disability from diseases, injuries, and risk factors in 1990 and projected to 2020. Harvard School of Public Health, Harvard.

M urray CJL et al. 1995. La carga global de enfermedad en 1990: resumen de los resultados, análisis de la sensibilidad y orientaciones futuras. Bolletin de la Oficina Sanitária Panamericana 118:510-528.

Nedel FB, Rocha M \& PereiraJ 1999. Anos de vida perdidos por mortalidade: um dos componentes da carga de doenças. Revista de Saúde Pública 33(5):460-469.

Nord E, Pinto-Prades JL, Richardson J, M enzel P \& Ubel PA 1999. Incorporating societal concerns for fairness in numerical valuations of health programmes. $\mathrm{H}$ ealth Economics 8:25-39.

Omram AR 2001. The epidemiologic transition: a theory of the epidemiology of population change. Bulletin of theWorld Health Organization 79(2):161-170.

Popkin BM 1994. The nutrition transition in low-income countries: an emerging crisis. Nutrition Reviews 52 (9):285-298.

Possas CA 2001. Social ecosystem health: Confronting the complexity and emergence of infecctious diseases. Cadernos de Saúde Pública 17:31-41.

Ramos LR, Rosa TEC, Oliveira ZM, M edina M CG \& Santos FRG 1993. Perfil do idoso em área metropolitana na região Sudeste do Brasil: resultados de inquérito domiciliar. Revista de Saúde Pública 27(2):87-94.

Santos-Preciado JI et al. 2003. La transición epidemiológica de las y los adolescentes em M éxico. Salud Pública de M éxico 45(supl 1):140-152.

Silva NAS \& M aldonado C 2003. A necessidade de mudança nas estratégias de intervenção para controlar a hipertensão arterial. Revista da SOCERJ 16(1):65-76.

World Health Organization 2003. Diet, nutrition and the prevention of chronic diseases. WHO Technical Report Series № 916: Geneva.

Artigo apresentado em 10/7/2004

Aprovado em 20/9/2004

Versão final apresentada em 25/9/2004 